\title{
Tracking the fate of glomerular epithelial cells in vivo using serial multiphoton imaging in novel mouse models with fluorescent lineage tags
}

\author{
Matthias J. Hackl ${ }^{1,2}$, James L. Burford ${ }^{1}$, Karie Villanueva ${ }^{1}$, Lisa Lam ${ }^{1}$, Katalin Suszták ${ }^{3}$, \\ Bernhard Schermer ${ }^{2}$, Thomas Benzing ${ }^{2}$, and János Peti-Peterdi ${ }^{1}$ \\ ${ }^{1}$ Departments of Physiology and Biophysics and Medicine, Zilkha Neurogenetic Institute, \\ University of Southern California, Los Angeles, CA \\ ${ }^{2}$ Department II of Internal Medicine and Center for Molecular Medicine Cologne, University of \\ Cologne, Cologne, Germany \\ ${ }^{3}$ Renal, Electrolyte and Hypertension Division, Department of Medicine, University of \\ Pennsylvania, Philadelphia, PA
}

\begin{abstract}
Podocytes are critical in the maintenance of a healthy glomerular filter, however they have been difficult to study in the intact kidney due to technical limitations. Here we report the development of serial multiphoton microscopy (MPM) of the same glomeruli over several days to visualize the motility of podocytes and parietal epithelial cells (PEC) in vivo. In Podocin-GFP mice podocytes formed sporadic multi-cellular clusters after unilateral ureteral ligation (UUO) and migrated into the parietal Bowman's capsule. The tracking of single cells in Podocin-confetti mice featuring cell-specific expression of CFP, GFP, YFP, or RFP revealed the simultaneous migration of multiple podocytes. In PEPCK-GFP mice serial MPM found PEC-to-podocyte migration and nanotubule connections. Our data support the highly dynamic rather than static nature of the glomerular environment and cellular composition. Future application of this new approach promises to advance our understanding of the mechanisms of glomerular injury and regeneration.
\end{abstract}

\section{Introduction}

Podocytes are critical elements of the glomerular filtration barrier (GFB), a complex vascular unit in the glomerular capillaries that performs plasma ultrafiltration ${ }^{1}$. Podocytes are highly differentiated cells which form long primary and secondary extensions that wrap around the outside of the capillary loops. Between their interdigitating foot processes the slit diaphragm is formed which is a key component of the $\mathrm{GFB}^{2}$. Recent genetic and cell

\footnotetext{
Users may view, print, copy, download and text and data- mine the content in such documents, for the purposes of academic research, subject always to the full Conditions of use: http://www.nature.com/authors/editorial_policies/license.html\#terms

Contributions

J.P-P. conceived the study. J.P-P., M.J.H., and J.L.B. designed the experiments, coordinated the study and analyzed the data. J.P-P., M.J.H. and T.B. wrote the manuscript. M.J.H., J.L.B., L.L., K.V., B.S. and K.S. performed the experiments.

Conflict of interest/disclosure

None of the authors has any conflicts of interest to declare.
} 
biological studies highlighted the critical importance of podocytes in the development of glomerular diseases ${ }^{3-11}$. Still, a critical barrier in understanding the mechanistic details of glomerular pathology is the technical limitation to study the GFB in its native environment. Due to the lack of in vivo data, there are still significant gaps in our understanding of podocyte dynamics and motility and their link to albuminuria and glomerulosclerosis. However, this insight would be needed for the development of new treatment strategies.

Because of the dynamic and complex structure of the GFB, the application of high resolution imaging tools may provide the long missing in vivo technology for podocyte research. MPM is a revolutionary, minimally invasive optical sectioning technique which allows the imaging of the mouse kidney ${ }^{12}$ including glomeruli in vivo ${ }^{13-15}$. Mouse genetic tools permit the cell-specific expression of multicolor fluorescent proteins ${ }^{16,17}$ including in podocytes for imaging applications ${ }^{18-22}$. Also, genetic tagging and lineage tracing are powerful tools to study cell fate in vivo. Therefore we aimed to develop a new visual approach to directly visualize and track the two glomerular epithelial cell types, podocytes and PECs in vivo in the intact mouse kidney using serial MPM over time.

The motility of podocytes and PECs and their role in renal pathologies and nephron regeneration are debated. According to the classic view podocytes are terminally differentiated cells and cannot repair themselves by means of cell division ${ }^{23,24}$. However, podocytes can proliferate in a limited number of conditions ${ }^{25}$. Lately the new research field of renal stem cells has also challenged the stationary podocyte concept by reports that podocytes can be replaced by PECs which migrate along the Bowman's capsule ${ }^{26}$ and express progenitor cell markers ${ }^{27}$. Visual techniques to track podocytes and PECs in vivo may aid research on these topics and the future development of novel progenitor or stem cell-based therapeutic approaches to renal injury. To test the utility of our new MPM imaging approach, two disease models were used: UUO and adriamycin nephropathy. UUO is a widely used animal model to study progressive renal disease and tubulointerstitial fibrosis $^{28}$. UUO pathology features injury of PECs and damage of the tubuloglomerular junction leading to the formation of atubular glomeruli ${ }^{29}$. The rapid remodeling of the Bowman's capsule observed after $\mathrm{UUO}^{29}$ suggested that this model may be ideally suited to image PEC and podocyte motility in kidney injury. Adriamycin nephropathy is a model resembling human focal segmental glomerulosclerosis ${ }^{30}$.

\section{Results}

\section{Characterization of Podocin-GFP (Pod-GFP) mice}

Previously we visualized podocytes based on their typical position and shape by a negative labeling technique in wild type animals using systemic injections of Lucifer Yellow, a freely filterable dye $\mathrm{e}^{14}$ (Supplementary Fig. 1a). However, this precluded their tracking during damage, migration or replacement. Therefore, we generated a Pod-GFP mouse model in which podocytes express membrane-targeted GFP, while all other cells express the red fluorescent protein Tomato. (Supplementary Fig. 1b). GFP fluorescence outlined a single cell layer and was most intense closely around the capillary loops compared to the cell body, due to the membrane-rich foot process region (Inset, Supplementary Fig. 1b). GFP expressing podocytes co-localized with cells identified by the negative labeling technique 
confirming the validity of our previous technique (Supplementary Fig. 1c, and

Supplementary Movie 1). Co-staining for GFP and synaptopodin, a podocyte differentiation marker, confirmed podocyte-specific expression of GFP (Supplementary Fig. 1d-f).

\section{MPM imaging of podocyte migration}

Compared to the lack of labeling in control wild-type mice (Fig. 1a) podocyte-specific GFP expression in Pod-GFP mice is demonstrated in a low-power MPM image of a kidney 3 weeks after UUO (Fig. 1b). Starting 1-2 weeks after UUO MPM imaging visualized podocytes that formed sporadic multi-cellular clusters on the glomerular tuft (Fig. 1c) with short projections developing towards the Bowman's space and into the remainder of the proximal tubule fragment (Fig. 1d). Occasionally, detached single podocytes were found further downstream in the lumen of proximal tubules in a few filtering nephrons, indicating podocyte shedding from these projections (Fig. 1e). However, the most common morphological change in the UUO model was the appearance of podocytes in the parietal layer of the Bowman's capsule. In most glomeruli podocyte projections propagated to the PEC layer after a contact between podocytes and PECs was established. These contact points developed either via continuous transition at the vascular pole (Fig. 1f) or by forming bridges across the Bowman's space close to the urinary pole (Supplementary Fig. 2a, and Supplementary Movie 2) or anywhere around the glomerular tuft (Fig. 1g). The podocyte projections appeared to grow further along the PEC layer towards the remodeled (sealed) glomerulotubular junction and in some glomeruli to the proximal tubule fragment (Supplementary Fig. 2a-c). Three to four weeks after UUO a continuous layer of GFPexpressing (GFP+) cells were found along the parietal Bowman's capsule and numerous cell bridges of podocyte origin were formed between the visceral and parietal cell layers (Fig. $1 \mathrm{~g}$ ). Statistical analysis (Fig. 1h) showed that $<5$ weeks after UUO the percentage of glomeruli in which $>50 \%$ of the parietal layer was covered by GFP+ cells was $19 \pm 4 \%$ (a total of $n=895$ glomeruli analyzed from $n=43$ mice) compared to $3 \pm 2 \%$ in age-matched control mice ( $\mathrm{n}=239$ glomeruli from $\mathrm{n}=19$ mice, $\mathrm{p}<0.05)$. The percentage of glomeruli with $>50 \%$ parietal GFP+ cell coverage increased continuously with time after UUO (Fig. 1i) $(\mathrm{R}=0.846, \mathrm{n}=48$ mice, $\mathrm{p}<0.05)$. Supplementary Fig. $2 \mathrm{~b}$ demonstrates the rare phenomenon of GFP+ cell projections along the parietal layer of Bowman's capsule in control healthy (nonUUO) kidneys. Importantly, we never observed solitaire or isolated clusters of GFP+ cells in the parietal layer. These cells always had continuous physical connections to the glomerular tuft suggesting that they originated from propagating visceral podocytes. Immunolabeling of GFP and the podocyte marker podocin 9 weeks after UUO found expression of podocin in GFP+ cells in the PEC layer, further suggesting the migration of podocytes in this model (Supplementary Fig. 2d-f).

To confirm that GFP+ cells in the parietal layer developed by the migration of visceral podocytes rather than podocin gene activation in PECs (hence Cre-mediated GFP expression), we repeated the experiment with tamoxifen-inducible Pod-GFP mice (iPodGFP mice). There was no GFP expression outside the visceral podocyte layer in iPod-GFP mice after tamoxifen induction (Fig. 1i, Fig. 2a). However, >2-4 weeks after UUO, iPodGFP mice developed the same glomerular phenotype as described above for the constitutive Pod-GFP model, including podocyte clustering (Fig. 2a), the development of visceral-to- 
parietal podocyte projections (bridging), and podocyte migration to the parietal layer (Fig. 2b-c). Statistical analysis (Fig. 1i) showed that similarly to the constitutive Pod-GFP model, the percentage of glomeruli with $>50 \%$ parietal GFP+ cell coverage in iPod-GFP mice increased continuously with aging after UUO $(\mathrm{R}=0.972, \mathrm{n}=296$ glomeruli analyzed from $\mathrm{n}=5$ mice, $\mathrm{p}<0.05$ ), although the phenomenon had apparently different dynamics in the two models.

In addition, to study the effects of a different injury, Pod-GFP mice were treated with a single high dose of adriamycin. Similarly to the UUO model, adriamycin treatment within 4 days induced the development of sporadic podocyte clusters (Fig. 2d, Supplementary Movie 3) and the appearance of GFP+ cells in the parietal layer close to the vascular pole transition, and in continuum with the visceral podocyte clusters (Fig. 2e). Histological analysis (Fig. 2f) revealed the significantly higher percentage of glomeruli with podocyte clusters 4 days after adriamycin treatment $(7 \pm 2 \%$, from a total of $n=1241$ glomeruli, analyzed from $n=4$ mice) compared to $0 \pm 0 \%$ in age-matched control mice ( $\mathrm{n}=1460$ glomeruli in $\mathrm{n}=4$ mice, $\mathrm{p}<0.05$ ). Albuminuria was measured in the same groups and confirmed that glomerular damage developed after adriamycin treatment (Supplementary Fig. 3).

\section{Serial in vivo MPM imaging of the same glomerulus}

To better understand the dynamics of podocyte migration we developed serial MPM imaging of the same glomerulus over time in the intact Pod-GFP mouse kidney in vivo, once in every 24 hours. Glomeruli were identified based on a laser-induced mark placed close to the glomerulus. Z-sectioning was performed at each time point and the z-stacks were compared afterwards. Serial MPM imaging was instrumental in depicting the dynamics and the dramatic changes in the morphology of podocyte projections which often occurred within 24 hours of the previous imaging session. We found evidence for the migration of podocytes away from the multi-cellular clusters, e.g. around the urinary pole into the remainder of the proximal tubule (Fig. 3a-b, Supplementary Movie 4). Also, the growth of GFP+ cell projections in the parietal layer was evident by the increasing length and width of the projections (Fig. 3c-d, Supplementary Movie 5). In the few nephrons which retained residual glomerular filtration, shedding of podocytes into the proximal tubule was observed (Supplementary Fig. 4).

\section{Tracking single podocytes in Podocin-Confetti (Pod-Confetti) mice}

By using Pod-Confetti mice with podocyte specific expression of either membrane-targeted CFP, nuclear GFP, cytosolic YFP or cytosolic RFP (Confetti construct ${ }^{17}$ ) and in vivo MPM imaging, we were able to identify and track over time individual podocytes based on their specific color (Fig. 4a and Supplementary Movie 6). As with Pod-GFP and iPod-GFP mice, UUO induced the bridging of podocytes over the Bowman's space to the parietal layer (Fig. 4b). Adjacent projections in the parietal layer were often labeled by a different color suggesting that multiple visceral podocytes migrate to the parietal Bowman's capsule. Statistical analysis of Confetti+ projections (Fig. 4c) showed that the majority of projections had either a visible, monochromatic bridge between the visceral and parietal layers or the parietal Confetti+ cell matched the color of the closest visceral podocyte $(81 \%$ in isolated projections, $73 \%$ in multiple projections). In addition, serial MPM imaging of the same 
glomerulus in the UUO kidney (Fig. 4d-e, Supplementary Movie 7) provided visual evidence for the appearance of new visceral podocytes within 24h. Also, MPM imaging of Pod-Confetti mice allowed the visualization of the interdigitating podocyte foot processes (Fig. 4f) as described in other genetic approaches ${ }^{18,19}$.

\section{MPM imaging of PEC migration}

To further elucidate the dynamic interaction between podocytes and PECs, we used in vivo serial MPM of PEPCK-GFP mice which express GFP in proximal tubule segments controlled by the PEPCK promoter ${ }^{31}$. MPM imaging of PEPCK-GFP mouse kidneys in vivo found strong GFP expression in some, but not all PECs in addition to proximal tubule cells, but not in the glomerular tuft (Fig. 5a). Immunofluorescence co-localization experiments confirmed GFP expression in some PECs (Supplementary Fig. 5). Occasionally we observed a GFP expressing PEC at the vascular pole of the glomerulus (Fig. 5b-c), which formed projections propagating into both the visceral and parietal layers (Supplementary Movie 8).

Interestingly, the strong GFP expression helped to visualize for the first time the presence of extremely thin nanotubules connecting PECs with the glomerular tuft after UUO (Fig. 5d). The width of these cell-to-cell connectors was uniform $(<350 \mathrm{~nm}$,) and they bridged over long distances between PECs and podocytes ( $>25 \mu \mathrm{m})$ across the entire Bowman's space. Nanotubule connections between PECs were also observed (not shown). The presence of PEC-to-podocyte nanotubules was quite common in the UUO model with an average of 1-4 per glomerulus. Time-lapse MPM imaging revealed (Supplementary Movie 9) that the nanotubules were steady over time regardless of variations in hemodynamics, suggesting that these structures are indeed nanotubules rather than long primary cilia. In addition, entire cell bodies appeared occasionally along the length of the nanotubules (Supplementary Fig. 2c) suggesting that nanotubules may participate in podocyte migration.

\section{Discussion}

Here we report the development of a new direct visual approach to track migrating podocytes and PECs over time in vivo in the same glomerulus of the intact kidney using serial MPM. This represents an important technical advance in nephrology research to study the dynamics and interactions of different glomerular cell types in the intact glomerular environment. The tracking of cell migration and alterations in glomerular morphology may be combined with quantitative MPM imaging of key glomerular functions as we reported recently ${ }^{32-34}$. The highly complex and dynamic portrayal of the structure and function of the same glomerulus during the course of a disease is one of the greatest values and advantages of this new MPM imaging technique. Accordingly, its first applications resulted in several new biological and anatomical discoveries. Serial MPM imaging provided visual evidence for podocyte motility and migration to the parietal Bowman's capsule in the intact mouse kidney in vivo, which were very robust after UUO. MPM imaging allowed us to visualize the development of multi-cellular podocyte clusters after UUO and adriamycin treatment, and the highly dynamic nature of the migration of the same podocytes and PECs over several days after UUO. Moreover, a new anatomical discovery is the first visualization of 
nanotubules connecting the visceral (podocytes) and parietal epithelial layers (PECs) which may participate in cell-to-cell communication and cell migration. The combination of serial MPM with mouse models, which allow the identification and tracking of single podocytes over time, will be very useful in future work to study mechanisms of glomerular disease and repair.

The feasibility of performing in vivo MPM imaging of the glomerulus in the intact mouse kidney was demonstrated before ${ }^{13-15,34}$, but this is the first study which utilized serial MPM in vivo to track over time the fate of podocytes and PECs that were individually marked using fluorescent lineage tags. Serial MPM complements the powerful classical genetic cell lineage tracing tools ${ }^{35}$ and can be combined with functional studies, e.g. measurement of the glomerular permeability of macromolecules as demonstrated by our lab recently ${ }^{32,33}$. Altogether, serial MPM is a significant new addition to the existing arsenal of tools to study podocytes and other glomerular cells ${ }^{14,18,34}$.

MPM imaging provided important visual clues on podocyte migration, consistent with the importance of podocyte motility and the intact actin cytoskeleton in GFB function as established by a number of recent studies ${ }^{3-11}$. Signs of podocyte clustering and/or migration were observed not only in the robust UUO model (Fig. 1-2c), but also in adriamycin nephropathy (Fig. 2d-f) and rarely in control kidneys (Supplementary Fig. 2b), suggesting that these are general phenomena not restricted to UUO, and may also be involved in the maintenance of the intact GFB. Serial MPM imaging over several days was instrumental to visualize the robust dynamics of podocyte and PEC migration and remodeling (Fig. 3-5, Supplementary Movies 4, 5, 7, 8). It should also be noted that protrusion and migration of these cluster cells into the proximal tubule occur in the absence of filtration (Fig. 3a-b), while podocyte detachment and shedding occur in the presence of mechanical forces (Fig. 1e, Supplementary Fig. 4). Based on the present findings, podocyte migration contributes to the pathological remodeling of the glomerulotubular junction and the Bowman's capsule after $\mathrm{UUO}^{29}$. Our data are consistent with the previously described presence of podocytes in the parietal Bowman's capsule in certain pathologies ${ }^{36,37}$ and rarely in the healthy kidney ${ }^{38}$.

With longer periods of UUO, a complete GFP+ cell lining of the parietal Bowman's capsule, and a progressive increase in the percentage of glomeruli with parietal GFP+ cell coverage were observed (Fig. 1g-i). These findings suggest that podocyte clustering and migration are constantly ongoing phenomena, at least in UUO. Although mice with inducible Cremediated GFP expression (iPod-GFP mice) showed the same general phenotype as the constitutive Pod-GFP model further confirming podocyte migration (Fig. 1-2), the parietal GFP+ cell coverage developed faster in Pod-GFP versus iPod-GFP mice (Fig. 1i). This was likely due to either imperfect Cre induction ${ }^{17}$ in iPod-GFP mice, or PEC-to-podocyte transformation in the constitutive Pod-GFP mice. Also, in Pod-Confetti mice with constitutive Cre expression it is possible that a change in podocyte color ("flipping") occurs even after the initial Cre recombination ${ }^{39}$. Therefore, the future use of inducible Cre models is required for cell lineage tracing experiments, while constitutive Cre reporter models may be used for short-term serial MPM imaging of cell migration. 
Another finding was the propagation of PECs observed in the PEPCK-GFP mouse model. We noticed Cre activity in some, but not all PECs in addition to the cells of the proximal tubule (Fig. 5a, Supplementary Fig. 5). Consistent with recent reports on the replacement of podocytes by $\mathrm{PECs}^{26,27}$ serial MPM imaging was able to visualize PEC propagation into both the visceral and parietal layers at the vascular pole of the glomerulus (Fig. 5b-c, Supplementary Movie 8).

The visualization of nanotubules interconnecting PECs and podocytes (Fig. 5d) is an exciting anatomical discovery that was made possible by the new highly fluorescent mouse models and the application of MPM in vivo. The (patho)physiological relevance of these physical connectors between PECs and podocytes that bridge over long distances within the Bowman's space is yet to be established. Nanotubules may provide an alternative to the vascular pole migration hypothesis put forward by recent reports ${ }^{26,27}$ and constitute a guiding structure for cell migration directly over the Bowman's space (Supplementary Fig. $2 \mathrm{c}$ ), or could be a unique form of cell-to-cell communication. Nanotubules were reported to allow the unidirectional transfer of specific molecules and organelles between cells ${ }^{40}$.

In conclusion, serial MPM imaging of the intact kidney in vivo using novel fluorescent lineage tagged mouse models is an exciting new tool to study the development of kidney disease. This imaging approach solves a critical technical barrier in podocyte research and allows the dynamic portrayal of the fate and function of various glomerular cell types in vivo. The results of the first applications of this technique may help to change current paradigms in glomerular biology and support the emerging new concept, which emphasizes the dynamic rather than static nature of podocytes, PECs and the entire glomerular environment. Serial MPM imaging has tremendous potential and utility in the future development and testing of new regenerative therapeutic approaches.

\section{Materials \& Methods}

\section{Animals}

Both male and female C57BL6/J mice at the age of 4-12 weeks were used randomly. Four new fluorescent reporter mouse models were generated: (i) Pod-GFP by crossing mice expressing Cre recombinase under the control of the podocin promoter ${ }^{21}$ and animals with a

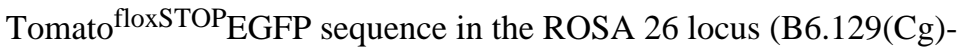
$G t\left(\text { ROSA)26Sor }{ }^{\text {tm } 4(A C T B-t d T o m a t o,-E G F P) L u o}\right)^{16}$. This results in an expression of Tomato in all cells except in podocytes, which express GFP. To study the effect of different backgrounds those mice were used in a C57BL6 background, a FvB background or the F1 generation crossed between these backgrounds. (ii) iPod-GFP by crossing mice expressing tamoxifeninducible improved Cre recombinase under the control of the podocin promoter (podocin$\mathrm{iCreER}^{\mathrm{T} 2}$ mice $^{22}$, kind gift from Dr. Farhad R. Danesh, Baylor College of Medicine) and animals with the Tomato ${ }^{\text {floxSTOP}}$ EGFP construct mentioned above. (iii) PEPCK-GFP by crossing mice expressing Cre recombinase under the control of the phosphoenolpyruvatecarboxykinase promoter (PEPCK-Cre, kind gift from Dr. Volker Haase, Vanderbilt University) $^{31}$ and animals with the Tomato ${ }^{\text {floxSTOP }}$ EGFP construct mentioned above. (iv) Pod-Confetti by crossing mice expressing the R26R-Confetti construct ${ }^{17}$ and podocin-Cre mice $^{21}$ resulting in the expression of either membrane-targeted CFP, nuclear GFP, cytosolic 
YFP or cytosolic RFP in podocytes. All animals were purchased from the Jackson laboratory (Bar Harbor, ME) or otherwise noted. All animal protocols were approved by the Institutional Animal Care and Use Committee at the University of Southern California or by local government authorities in Germany (LANUV NRW) under the license 8.8750.10.31.08.049.

\section{Tamoxifen induction}

4 weeks-old mice received Tamoxifen chow ( $40 \mathrm{mg} / \mathrm{kg}$ body weight, Harlan Laboratories, Indianapolis, IN) for 2 weeks, followed by a 2 week washout period before additional procedures were performed.

\section{UUO}

Between 3 and 8 weeks of age the animals were anaesthetized with isoflurane, and after a midline laparotomy the left ureter was exposed and ligated three times. Successful ligation was confirmed by the hydronephrotic distension of the kidney at the time of imaging.

\section{Adriamycin nephropathy}

Pod-GFP animals received a single dose of $25 \mathrm{mg} / \mathrm{kg}$ Adriamycin (Doxorubicin) via retroorbital injection and were imaged, sacrificed and their tissues collected 4-6 days later. Successful induction of albuminuria was confirmed by measuring the urinary albumin/ creatinine ratio (Exocell, Philadelphia, PA) between days 0-6 after adriamycin treatment.

\section{Multiphoton imaging}

The animals were anaesthetized with a combination of ketamine $(100 \mathrm{mg} / \mathrm{kg})$ and xylazine $(10 \mathrm{mg} / \mathrm{kg})$. A tracheal tube was placed to facilitate breathing and the right carotid artery and/or jugular vein was cannulated for dye infusion. A 70kDa Texas red dextran or Alexa 594 bovine serum albumin was injected to label the vasculature. In some experiments Lucifer Yellow (Invitrogen) was injected as iv bolus to visualize glomerular filtration. The left kidney was exteriorized through a flank incision and the animal placed on the microscope stage as described before ${ }^{34}$. Body temperature was maintained with a homeothermic blanket system (Harvard Apparatus). The images were acquired using a Leica TCS SP5 multiphoton confocal fluorescence imaging system with a 63x Leica glycerineimmersion objective (NA 1.3) powered by a Chameleon Ultra-II MP laser at $860 \mathrm{~nm}$ (Coherent) and a DMI 6000 inverted microscope's external nondescanned detectors. Short pass filters (680 $\mathrm{nm}$ for blue and red, $700 \mathrm{~nm}$ for green and yellow), dichroic mirrors (cut off at $515 \mathrm{~nm}$ for green and yellow, $560 \mathrm{~nm}$ for blue and red) and bandpass filters were specific for detecting CFP/GFP/YFP/RFP emission ( $473 \mathrm{~nm} / 514 \mathrm{~nm} / 545 \mathrm{~nm} / 585 \mathrm{~nm})($ Chroma). The images of iPod-GFP animals were acquired using the external detectors of an inverted Zeiss LSM710 NLO multiphoton confocal fluorescence microscope powered by a Chameleon Ultra-II MP laser at $860 \mathrm{~nm}$ and a 40x Zeiss water-immersion objective (NA1.2).

\section{Serial survival imaging of the same glomerulus}

After anaesthesia the left kidney was exteriorized via a small cut in the left flank below the kidney to avoid sutures right above the kidney afterwards. To avoid invasive vascular access 
surgeries, the dyes were administered by retroorbital injections. The animal was transferred to the microscope stage and the exteriorized kidney was placed into a kidney cup. An area of the kidney suitable for imaging was identified and the position of the kidney was noted for identical placement on the following days. After acquiring z-stacks of the glomerulus a small distant area in the field of view was marked by shortly focusing the laser beam on this area with high power. This maneuver generated an easy to find highly fluorescent spot (reference point) which remained there for 3-5 days. The position of the mark relative to the glomerulus of interest was documented. After imaging, the kidney was placed back into the retroperitoneum and the flank cut was closed with two layers of sutures. This procedure was repeated 24, and 48 hours later by removing the sutures and exteriorizing the kidney again. With this technique we were able to subsequently find approximately $70 \%$ of the glomeruli that were marked in the first imaging session. Z-stacks of marked glomeruli were acquired with identical imaging settings as the day before.

The potential toxicity of laser excitation and fluorescence to the cells were minimized by using a low laser power and high scan speeds to keep total laser exposure as small as possible. The usual image acquisition consisted of only one $\mathrm{z}$-stack per glomerulus $(<1$ minute) per 24 hours which resulted in no apparent cell injury. Serial imaging once every 24 hours for up to 72 hours after the first imaging session and the associated multiple, dorsal abdominal surgeries did not cause visible tissue adhesions or renal fibrosis.

\section{Z-stack analysis}

Z-stacks from different time points were aligned using StereoMovie Maker (http:// stereo.jpn.org) to visualize changes over time in a side-by-side fashion, see Supplementary movies.

\section{Immunohistochemistry}

Animals were perfused with ice cold PBS into the left ventricle followed by ice cold $4 \%$ PFA for 2 minutes each. Antigen retrieval was performed on paraffin sections with a heating step for $8 \mathrm{~min}$ at $95^{\circ} \mathrm{C}$ in a citrate buffer using a pressure cooker. The sections were incubated with the following primary antibodies (1:100 dilution unless stated otherwise) overnight: anti-synaptopodin (mouse, Progen, Heidelberg, Germany), anti-podocin (1:250, rabbit, Sigma-Aldrich, St. Louis, MO), anti-claudin-1 (1:250, rabbit, Sigma), anti-villin (mouse, Immunotech, Chicago, IL), anti-GFP (chicken, Aves, Tigard, OR) and anti-GFP (rabbit, Invitrogen) followed by incubation with the secondary antibodies conjugated with Alexa 488/594. Confocal fluorescence microscopy was performed using the same Leica TCS SP5 microscope as above.

\section{Statistical analysis}

Data are expressed as average \pm s.e.m. and were analyzed non-blinded using Student's t-test or linear regression ( $\mathrm{R}$ value) as indicated. $\mathrm{P}<0.05$ was considered significant. For the statistical analysis of visceral to parietal projections in Confetti-UUO mice, multiple projections were defined as projections in which parietal Confetti+ cells had direct contact with other projections ( $n=167$ isolated projections, $n=217$ multiple projections, from a total of $\mathrm{n}=112$ glomeruli analyzed from 4 mice). 


\section{Supplementary Material}

Refer to Web version on PubMed Central for supplementary material.

\section{Acknowledgments}

This work was supported in part by the US National Institutes of Health grant DK64324, by the American Diabetes Association grant 1-11-BS-121, by the University Kidney Research Organization to J.P-P, by Deutsche Forschungsgemeinschaft SFB635 to T.B, and by US National Institutes of Health grant DK076077 to K.S. M.J.H. was supported by Deutsche Forschungsgemeinschaft grant HA-6212. We thank Matthias Hammerschmidt and Hans-Martin Pogoda (Institute of Developmental Biology, University of Cologne) for granting access to their imaging facility.

\section{References}

1. Patrakka J, Tryggvason K. New insights into the role of podocytes in proteinuria. Nat Rev Nephrol. 2009; 5:463-468. [PubMed: 19581907]

2. Pavenstädt H, Kriz W, Kretzler M. Cell Biology of the Glomerular Podocyte. Physiol Rev. 2003; 83:253-307. [PubMed: 12506131]

3. Boute N, et al. NPHS2, encoding the glomerular protein podocin, is mutated in autosomal recessive steroid-resistant nephrotic syndrome. Nat Genet. 2000; 24:349-354. [PubMed: 10742096]

4. Huber TB, et al. Bigenic mouse models of focal segmental glomerulosclerosis involving pairwise interaction of CD2AP, Fyn, and synaptopodin. J Clin Invest. 2006; 116:1337-1345. [PubMed: 16628251]

5. Jones N, et al. Nck adaptor proteins link nephrin to the actin cytoskeleton of kidney podocytes. Nature. 2006; 440:818-823. [PubMed: 16525419]

6. Kaplan JM, et al. Mutations in ACTN4, encoding [alpha]-actinin-4, cause familial focal segmental glomerulosclerosis. Nat Genet. 2000; 24:251-256. [PubMed: 10700177]

7. Kestilä M, et al. Positionally Cloned Gene for a Novel Glomerular Protein-Nephrin-Is Mutated in Congenital Nephrotic Syndrome. Mol Cell. 1998; 1:575-582. [PubMed: 9660941]

8. Mundel P, Reiser J. Proteinuria: an enzymatic disease of the podocyte? Kidney Int. 2010; 77:571580. [PubMed: 19924101]

9. Reiser J, et al. Podocyte Migration during Nephrotic Syndrome Requires a Coordinated Interplay between Cathepsin L and a3 Integrin. J Biol Chem. 2004; 279:34827-34832. [PubMed: 15197181]

10. Shih N-Y, et al. Congenital Nephrotic Syndrome in Mice Lacking CD2-Associated Protein. Science. 1999; 286:312-315. [PubMed: 10514378]

11. Winn MP, et al. A Mutation in the TRPC6 Cation Channel Causes Familial Focal Segmental Glomerulosclerosis. Science. 2005; 308:1801-1804. [PubMed: 15879175]

12. Peti-Peterdi J, Burford JL, Hackl MJ. The first decade of using multiphoton microscopy for highpower kidney imaging. Am J Physiol Renal Physiol. 2012; 302:F227-F233. [PubMed: 22031850]

13. Devi S, et al. Multiphoton imaging reveals a new leukocyte recruitment paradigm in the glomerulus. Nat Med. 2013; 19:107-112. [PubMed: 23242472]

14. Peti-Peterdi J, Sipos A. A high-powered view of the filtration barrier. J Am Soc Nephrol. 2010; 21:1835-1841. [PubMed: 20576805]

15. Schiessl IM, Bardehle S, Castrop H. Superficial nephrons in BALB/c and C57BL/6 mice facilitate in vivo multiphoton microscopy of the kidney. PLoS One. 2013; 8:e52499. [PubMed: 23349687]

16. Muzumdar MD, Tasic B, Miyamichi K, Li L, Luo L. A global double-fluorescent Cre reporter mouse. Genesis (New York NY 2000). 2007; 45:593-605.

17. Snippert HJ, et al. Intestinal crypt homeostasis results from neutral competition between symmetrically dividing Lgr5 stem cells. Cell. 2010; 143:134-144. [PubMed: 20887898]

18. Grgic I, et al. Imaging of Podocyte Foot Processes by Fluorescence Microscopy. J Am Soc Nephrol. 2012

19. Höhne M, et al. Light Microscopic Visualization of Podocyte Ultrastructure Demonstrates Oscillating Glomerular Contractions. Am J Pathol. 2013; 182:332-338. [PubMed: 23246153] 
20. Khoury CC, et al. Visualizing the mouse podocyte with multiphoton microscopy. Biochem Biophys Res Commun. 2012; 427:525-530. [PubMed: 23022193]

21. Moeller MJ, Sanden SK, Soofi A, Wiggins RC, Holzman LB. Podocyte-specific expression of cre recombinase in transgenic mice. Genesis (New York NY 2000). 2003; 35:39-42.

22. Wang J, et al. Tamoxifen-inducible podocyte-specific iCre recombinase transgenic mouse provides a simple approach for modulation of podocytes in vivo. Genesis. 2010; 48:446-451. [PubMed: 20641128]

23. Shankland SJ. The podocyte's response to injury: Role in proteinuria and glomerulosclerosis. Kidney Int. 2006; 69:2131-2147. [PubMed: 16688120]

24. D'Agati VD, Kaskel FJ, Falk RJ. Focal segmental glomerulosclerosis. N Engl J Med. 2011; 365:2398-2411. [PubMed: 22187987]

25. Moeller MJ, et al. Podocytes Populate Cellular Crescents in a Murine Model of Inflammatory Glomerulonephritis. J Am Soc Nephrol. 2004; 15:61-67. [PubMed: 14694158]

26. Appel D, et al. Recruitment of podocytes from glomerular parietal epithelial cells. J Am Soc Nephrol. 2009; 20:333-343. [PubMed: 19092119]

27. Ronconi E, et al. Regeneration of glomerular podocytes by human renal progenitors. J Am Soc Nephrol. 2009; 20:322-332. [PubMed: 19092120]

28. Chevalier RL, Forbes MS, Thornhill BA. Ureteral obstruction as a model of renal interstitial fibrosis and obstructive nephropathy. Kidney Int. 2009; 75:1145-1152. [PubMed: 19340094]

29. Forbes MS, Thornhill BA, Chevalier RL. Proximal tubular injury and rapid formation of atubular glomeruli in mice with unilateral ureteral obstruction: a new look at an old model. Am J Physiol Renal Physiol. 2011; 301:F110-F117. [PubMed: 21429968]

30. Dai C, Saleem MA, Holzman LB, Mathieson P, Liu Y. Hepatocyte growth factor signaling ameliorates podocyte injury and proteinuria. Kidney Int. 2010; 77:962-973. [PubMed: 20375988]

31. Rankin EB, Tomaszewski JE, Haase VH. Renal cyst development in mice with conditional inactivation of the von Hippel-Lindau tumor suppressor. Cancer Res. 2006; 66:2576-2583. [PubMed: 16510575]

32. Nakano D, et al. Multiphoton Imaging of the Glomerular Permeability of Angiotensinogen. J Am Soc Nephrol. 2012; 23:1847-1856. [PubMed: 22997258]

33. Salmon AHJ, et al. Loss of the Endothelial Glycocalyx Links Albuminuria and Vascular Dysfunction. J Am Soc Nephrol. 2012; 23:1339-1350. [PubMed: 22797190]

34. Kang JJ, Toma I, Sipos A, McCulloch F, Peti-Peterdi J. Quantitative imaging of basic functions in renal (patho)physiology. Am J Physiol Renal Physiol. 2006; 291:F495-F502. [PubMed: 16609147]

35. Smeets B, et al. Tracing the Origin of Glomerular Extracapillary Lesions from Parietal Epithelial Cells. J Am Soc Nephrol. 2009; 20:2604-2615. [PubMed: 19917779]

36. Benigni A, et al. Inhibiting angiotensin-converting enzyme promotes renal repair by limiting progenitor cell proliferation and restoring the glomerular architecture. Am J Pathol. 2011; 179:628-638. [PubMed: 21718676]

37. Gibson IW, Downie TT, More IA, Lindop GB. Atubular glomeruli and glomerular cysts--a possible pathway for nephron loss in the human kidney? J Pathol. 1996; 179:421-426. [PubMed: 8869291]

38. Bariety J, Mandet C, Hill GS, Bruneval P. Parietal podocytes in normal human glomeruli. J Am Soc Nephrol. 2006; 17:2770-2780. [PubMed: 16943305]

39. Schepers AG, et al. Lineage Tracing Reveals Lgr5+ Stem Cell Activity in Mouse Intestinal Adenomas. Science. 2012; 337:730-735. [PubMed: 22855427]

40. Rustom A, Saffrich R, Markovic I, Walther P, Gerdes H-H. Nanotubular highways for intercellular organelle transport. Science (New York NY). 2004; 303:1007-1010. 

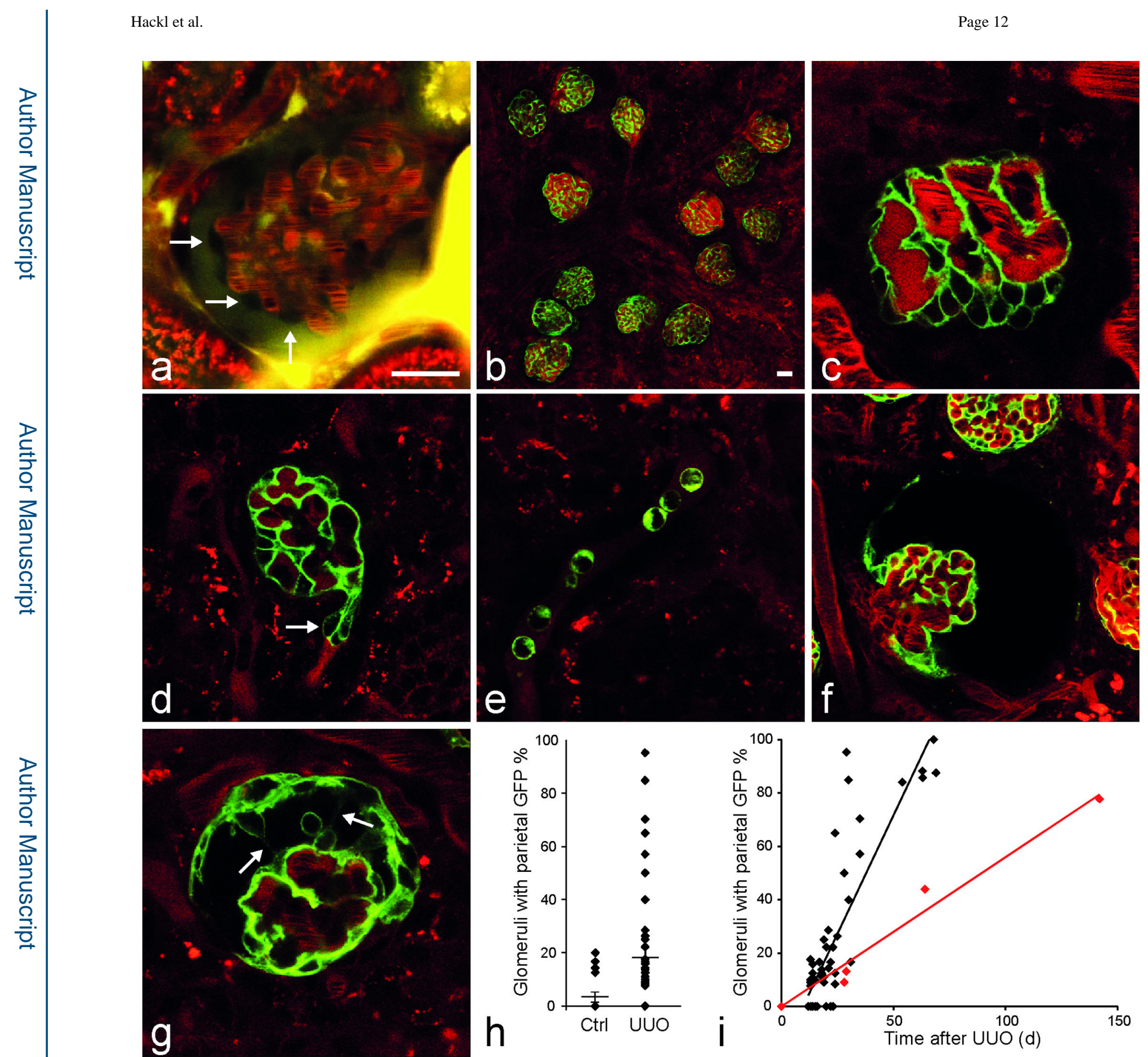

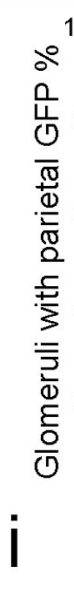

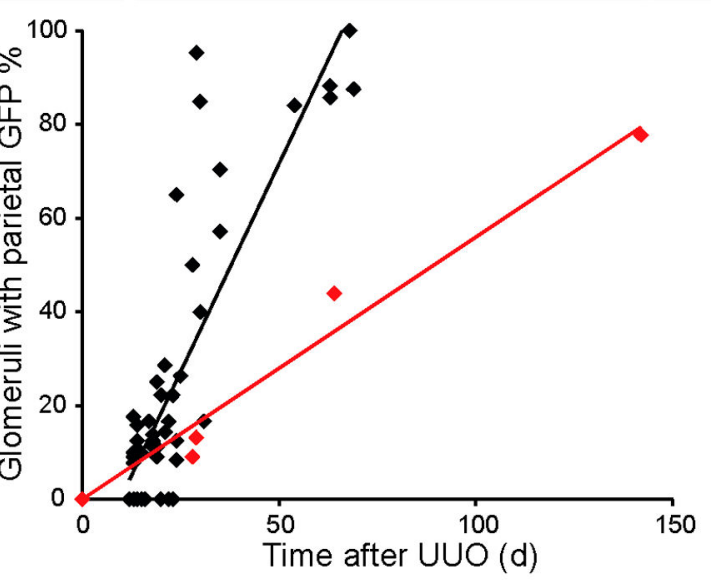

Figure 1. MPM imaging in vivo reveals signs of podocyte migration in the intact kidney in the model of unilateral ureteral obstruction (UUO) in Pod-GFP mice

(a) In a wild-type mouse kidney the glomerular podocytes appear as dark, unlabeled cells around capillaries (arrows). Plasma is labeled red with Alexa594-Albumin. Lucifer yellow (yellow) was injected iv to label the filtration (Bowman's) space. (b) Low-power image of glomeruli after UUO shows restriction of GFP fluorescence to podocytes in Pod-GFP mice. Early podocyte changes that appear after UUO include the development of podocyte cell clusters (c) and their projections protruding (arrow) into the lumen of the proximal tubule (d). (e) Detached, individual podocytes can be seen in the lumen of the proximal tubule. 
GFP+ cells in the parietal layer appear to develop by visceral podocytes migrating to the parietal layer via the vascular pole transition (f) or directly over the Bowman's space. (g) Thin connections appear to bridge the parietal and visceral layers of the Bowman's capsule (arrows). Continuous GFP+ cell coverage in the parietal layer (g) develops later, >3-4 weeks after UUO. Scale bars are $20 \mu \mathrm{m}$. Magnification is the same on all panels except panel b. (h) Percentage of glomeruli in which $>50 \%$ of the parietal layer is covered by GFP+ cells in age-matched control mice $(n=19)$ and $2-5$ weeks after UUO $(n=43)$. *: $P<0.05$. Data are presented as mean \pm s.e.m. (i) Linear fit of data points (Pod-GFP in black ( $\mathrm{n}=48$ mice), iPod-GFP in red ( $\mathrm{n}=5$ mice) shows the percentage of glomeruli with $>50 \%$ parietal GFP+ cell coverage. 

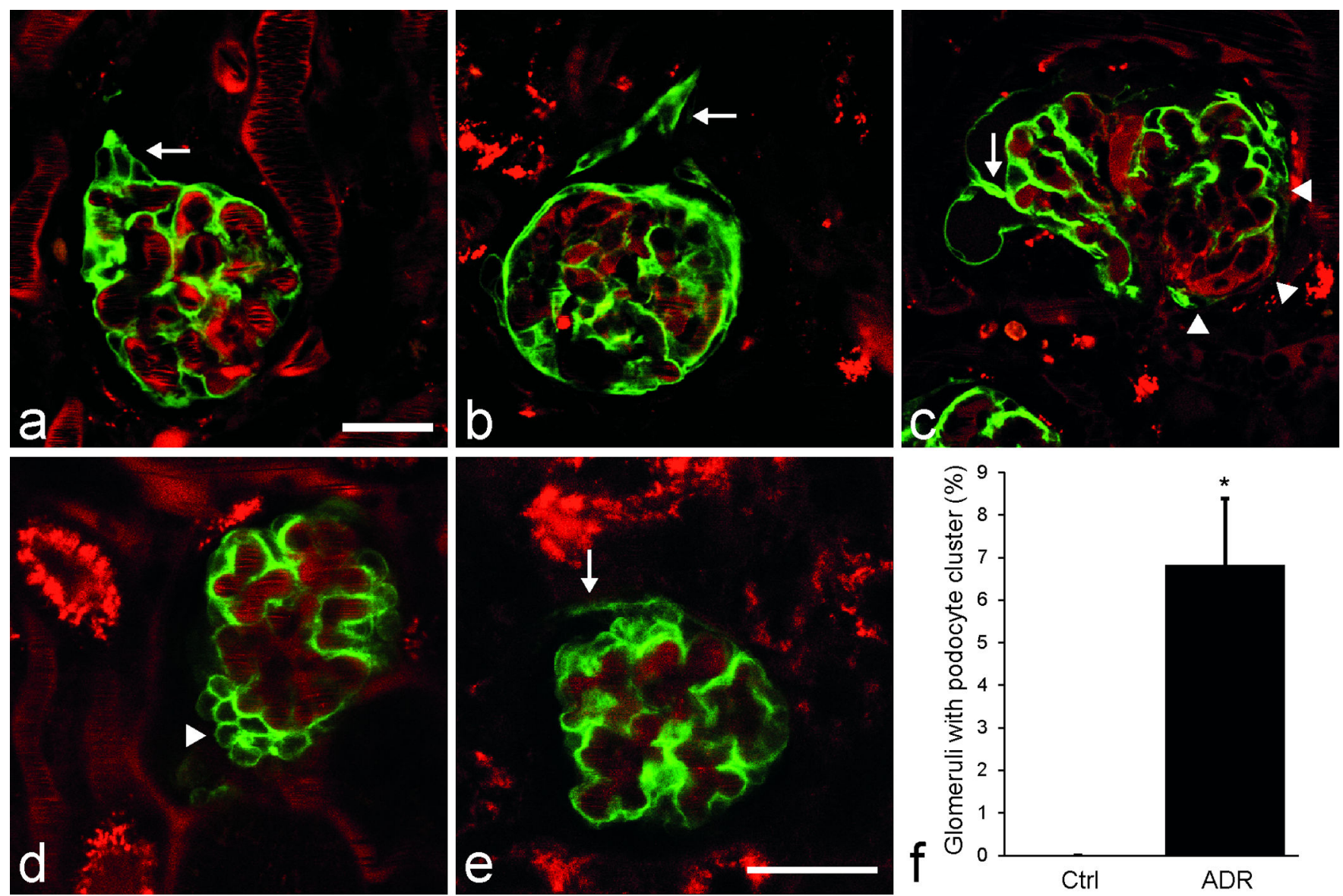

Figure 2. Confirmation of podocyte clustering and migration in iPod-GFP mice after UUO (a-c) and podocyte clustering in the model of adriamycin nephropathy in Pod-GFP mice (d-f)

In mice with tamoxifen-induced podocyte GFP expression (iPod-GFP) podocyte clustering was observed $<2$ weeks after UUO (a, arrow). After $>4$ weeks following UUO, podocyte projections to the parietal Bowman's capsule developed either at the urinary pole invading the glomerulo-tubular junction (b, arrow), or at the vascular pole transition (c, arrowheads), or anywhere in between the poles (c, arrow) giving rise to parietal GFP+ cells. Plasma was labeled red with Alexa594-Albumin. MPM imaging of the intact Pod-GFP mouse kidney after adriamycin treatment revealed the presence of podocyte clusters around capillaries (d, arrowhead) and the development of GFP+ cell coverage in the parietal layer which was continuous with the podocyte clusters via the vascular pole transition (e, arrow). Scale bar is $20 \mu \mathrm{m}$ for all panels. (f) Summary of histological analysis showing the percentage of glomeruli with podocyte clusters in age-matched control mice and 4 days after adriamycin treatment $(\mathrm{n}=4$ each). $*$ : $\mathrm{P}<0.05$. Data are presented as mean \pm s.e.m. 

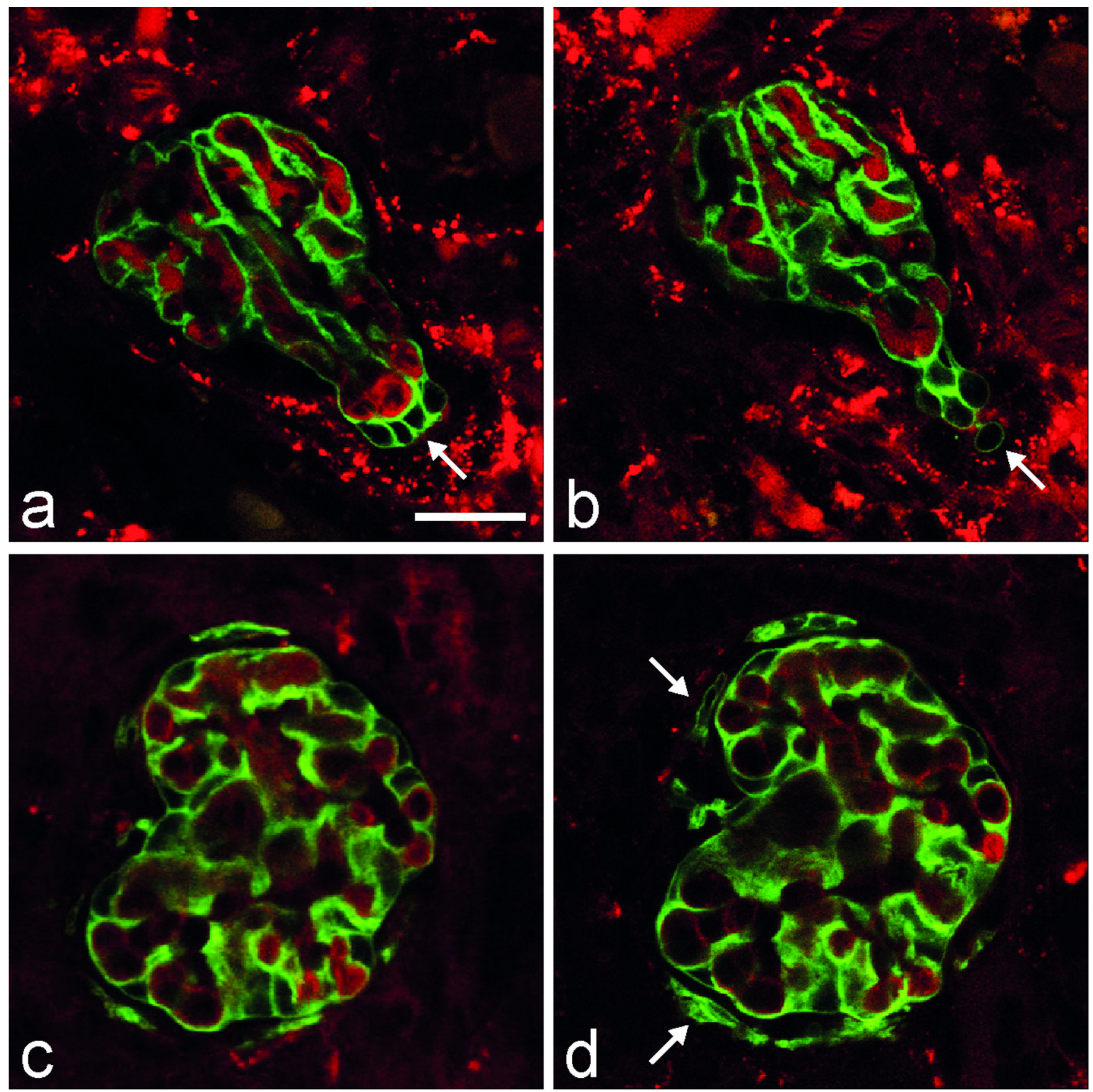

Figure 3. Serial in vivo MPM imaging of the same glomerulus in Pod-GFP mice after UUO over time, once in 24 hours

(a-b) Podocytes from a hypercellular area at the urinary pole of a collapsed, non-filtering glomerulus (a, arrow) appear to migrate away from the capillary tuft to form a projection into the remainder of the proximal tubule (b, arrow, $24 \mathrm{~h}$ later). (c-d) Demonstration of the increased size (growth) of the GFP+ cell projections in the parietal layer (d, arrows) 24 hours after the previous imaging session (c). Plasma is labeled red with Alexa594-Albumin. Scale bar is $20 \mu \mathrm{m}$ for all panels. 

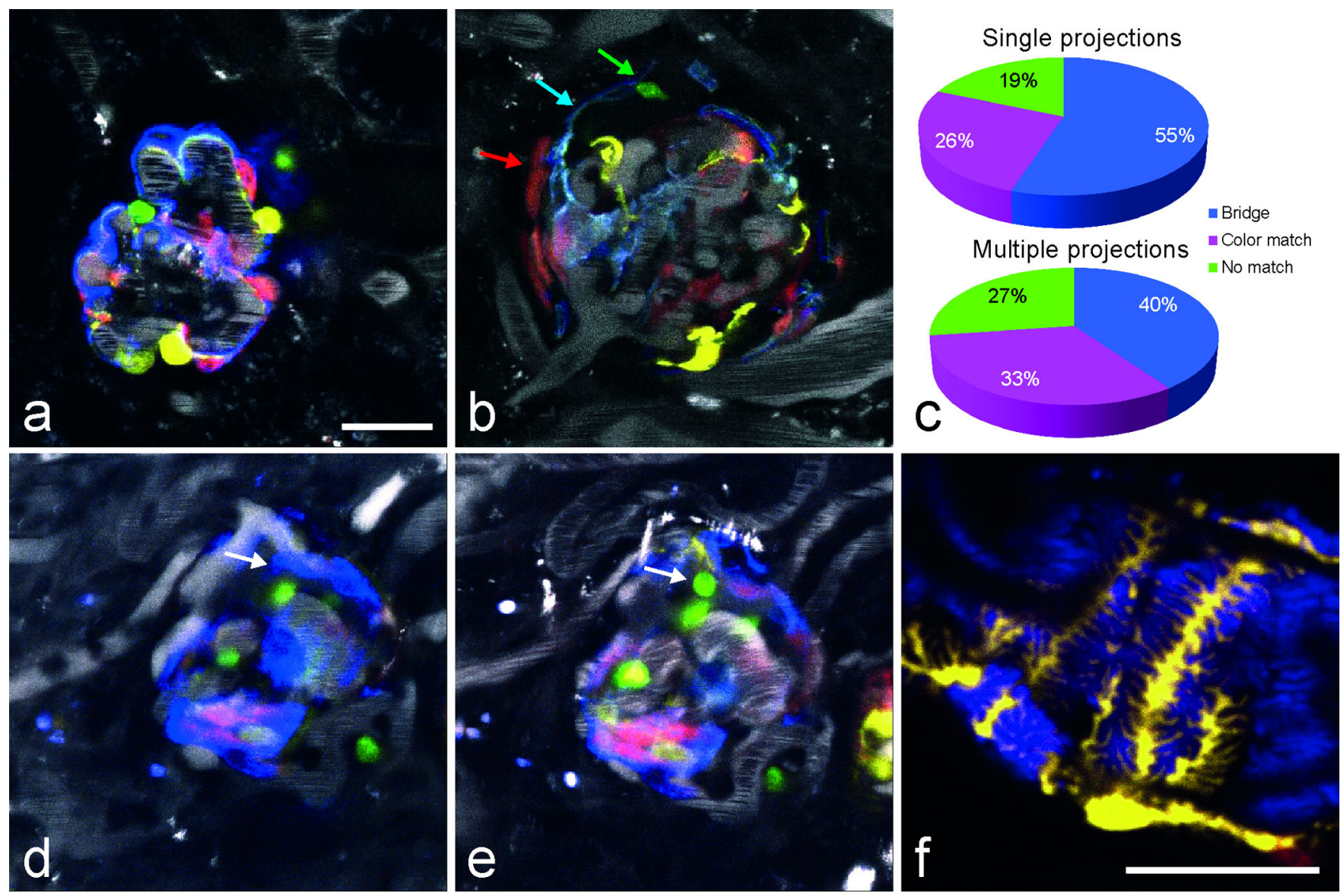

Figure 4. Identification and tracking of single podocytes in the multi-color Pod-Confetti mouse model using in vivo MPM imaging

(a) Podocytes are labeled in one of the four colors, either by membrane-targeted CFP, nuclear GFP, cytosolic YFP or cytosolic RFP. Labeled dextran (plasma dye) is shown in grayscale. (b) A CFP-labeled (blue) visceral podocyte is shown bridging over the Bowman's space to the parietal layer and in direct contact with other parietal cells of the same color (blue arrow). Other adjacent parietal Confetti+ cells are labeled with RFP (red arrow) or GFP (green arrow). (c) Analysis of the Confetti+ cell projections between the visceral and parietal layers shown for both single, isolated projections and for multiple projections in which parietal Confetti+ cells had direct contact with other projections $(n=167$ single and $\mathrm{n}=217$ multiple projections from 112 glomeruli in 4 mice). (d-e) Serial MPM imaging of the same glomerulus shows the appearance of a nuclear GFP-labeled podocyte around a glomerular capillary (e) compared to $24 \mathrm{~h}$ prior (d). Arrows indicate the same glomerular tuft region). d-e are projection images of 8 different confocal z-planes. (f) Cell morphology of podocytes including the interdigitating foot processes between two adjacent CFP and YFPlabeled podocytes are visualized in great detail (fixed, frozen tissue section). Scale bar is $20 \mu \mathrm{m}$ for all panels except panel $\mathrm{f}(1 \mu \mathrm{m})$. 

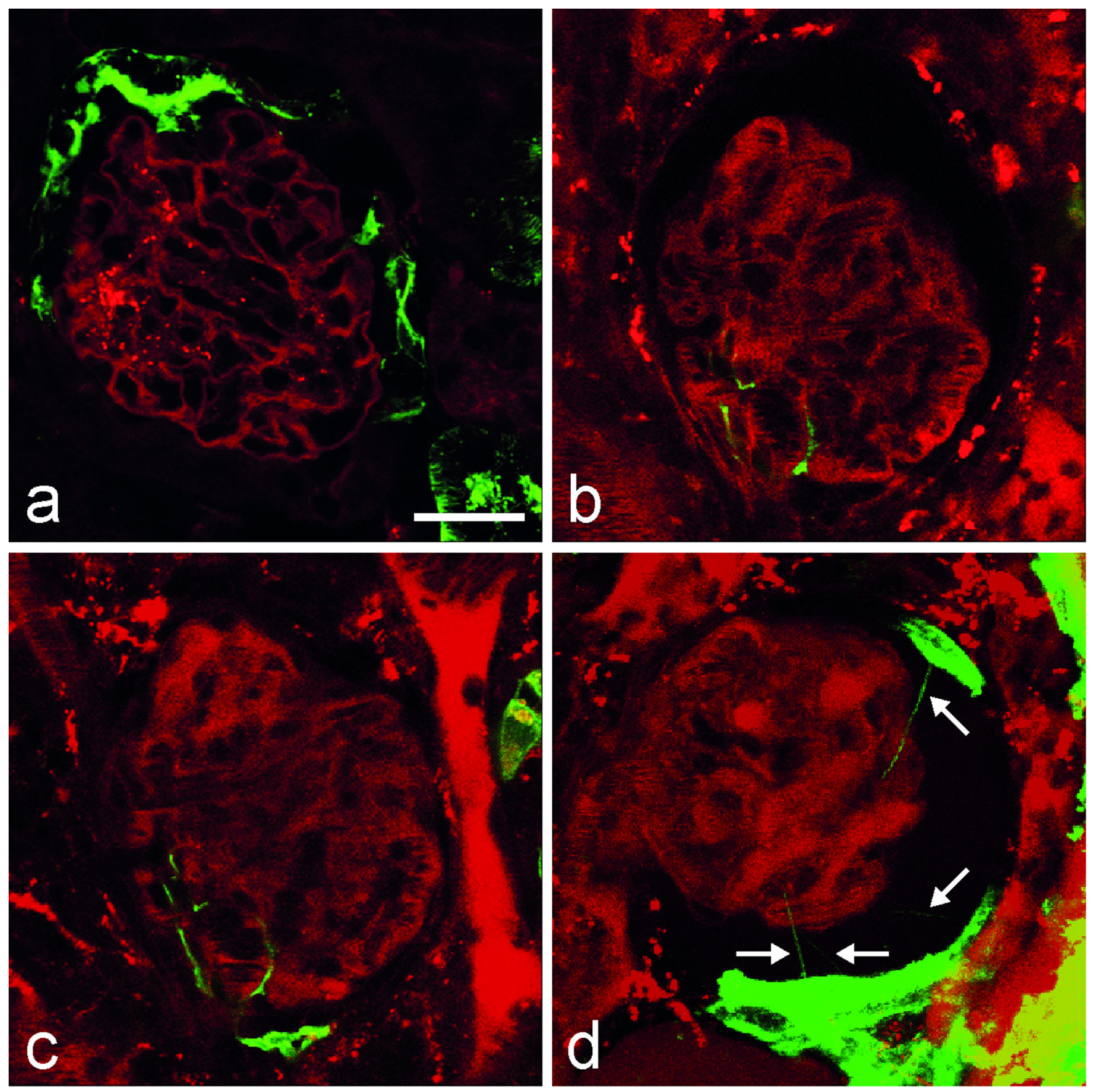

Figure 5. MPM imaging of PEC migration in vivo in the PEPCK-GFP mouse model after UUO (a) Cell-specific GFP expression in the proximal tubule and in some, but not all PECs. All other cells are labeled with Tomato. (b-c) Serial MPM imaging of the same glomerulus shows a GFP expressing PEC at the vascular pole of the glomerulus that forms projections propagating into both the visceral and parietal layers. The length of the green projections increased between the time points of $0 \mathrm{~h}(\mathrm{~b})$, and $48 \mathrm{~h}$ (c). (d) Four green nanotubules 
(arrows) are visualized in the intact glomerulus, which appear to connect the PEC layer with the glomerular tuft. Scale bar is $20 \mu \mathrm{m}$ for all panels. 